\title{
Spatial multiplexing for outdoor MIMO-OFDM systems with limited feedback costraint
}

\author{
S. Savazzi ${ }^{1}$, M. Nicoli ${ }^{1}$, M. Sternad ${ }^{2}$ \\ ${ }^{1}$ Dip. di Elettronica e Informazione, Politecnico di Milano, Piazza L. da Vinci 32, I-20133 Milano, Italy \\ ${ }^{2}$ Signals and Systems, Dept. of Engineering Sciences, Uppsala University, SE-75120 Uppsala, Sweden \\ e-mail: savazzi@elet.polimi.it, nicoli@elet.polimi.it,ms@signal.uu.se
}

\begin{abstract}
In this paper we propose a spatial multiplexing technique for the downlink of a multiple-input-multiple-output (MIMO) orthogonal-frequency-division-multiplexing (OFDM) system. For outdoor environments with a limited angular spread at the base station, the proposed technique is able to separate the users' streams through a joint spatial processing at both the transmitter and the receiver requiring only a limited feedback from each user. Adaptive transmission is adopted on each stream to set a fixed probability of error. Numerical simulations show that the proposed technique is able to provide significant throughput gains compared to fixed-beams based approaches proposed in the literature.
\end{abstract}

\section{INTRODUCTION}

Next generation mobile communication systems are expected to place stringent demands for high quality and high data rates over mobile radio channels. Recent studies on multiple input multiple output (MIMO) antenna systems with orthogonal frequency division multiplexing (OFDM) signaling have shown that spatial-frequency parallel processing at both transmitter and receiver, combined with fast adaptive transmission, is able to provide significant increases in spectral efficiency [1].

The use of multiple antennas enables also space-division multiple access (SDMA), which allows intra-cell bandwidth reuse by creating spatially multiplexed channels [2]. A fundamental challenge to be overcome is how the scheduler should separate and group the users: recent studies [3] focused on the uplink case and suggested clustering of users based on their mutual spatial correlation, or alternatively to their main direction of arrival (DOA) in case of moderate angular spread at the base station (BS). If channel state information (CSI) is perfectly known to the transmitter then interferencefree spatially multiplexed channels could be set up through appropriate precoding matrices or vectors [4]. However, for frequency division duplex (FDD) systems, this assumption leads to an unacceptable feedback rate requirement.

In this paper we focus on spatial multiplexing for the downlink of a multi-user FDD MIMO-OFDM system. A transmission strategy is designed to maximize the overall throughput under the constraint of a limited CSI at the BS. The proposed work is suited for outdoor environments where the BS is located higher than the surrounding scatterers so

This work was partially developed within the IST-FP6 Network of Excellence in Wireless Communications (NEWCOM). that transmitted signals propagate through a multipath channel with moderate angular spread [5], [6]. For the allocation of a specific time-frequency resource unit, a scheduler at the BS exploits quantized partial CSI on all the active users in order to maximize the cell throughput at a given limited feedback rate and minimize the multi-user interference (MUI) within the unit.

Unlike other general limited feedback oriented approaches [7], where the receiver chooses the precoding vectors from a finite codebook or grid [8] of fixed beams and then conveys the precoder selection to the transmitter using a limited number of bits, here a more flexible solution is proposed. The scheduler is in charge of adaptively selecting the best subset of users and designing the precoders based on the following CSI: an estimate of the main direction of departure (DOD) and a measurement of the signal to interference noise ratio (SINR) for each active user. An adaptive grid of beams (AGoB) is thus designed according to users' CSI, while at the receiver side a minimum variance distortionless response (MVDR) spatial filter is employed to maximize the received SINR.

The structure of the paper is as follows. The problem is outlined in Section II. Section III and IV deal with the proposed structure for the BS and the MS respectively. Performance analysis and a comparison between our $\mathrm{AGoB}$ solution and the so called fixed Grid of Beams (GoB) transmission scheme (D2.7 from [8]) is carried out through numerical simulations in Section V.

\section{PRoblem Definition AND SyStem MODEL}

We consider the downlink channel of a multiuser MIMOOFDM wireless system, where $L$ active mobile stations (MS) share the same cell. The BS is equipped with a uniform antenna array with $N_{\mathrm{T}} \Delta$-spaced elements, while each MS has $N_{\mathrm{R}}$ receiving antennas (for simplicity $N_{\mathrm{R}} \leq N_{\mathrm{T}}$ ). FDD is used to separate uplink and downlink communications.

In the system under study multiple access is handled by a combination of time, frequency and space division. As shown in Fig. 1, the OFDM channel is indeed organized in timefrequency resource units, each consisting of a frame of $W$ subsequent bursts of $D$ OFDM symbols and a bin of $B$ adjacent $\Delta f$-spaced subcarriers. The same time-frequency unit is allocated to a subset $\mathcal{S}$ of $M \leq N_{\mathrm{T}}$ users separated by means of spatial multiplexing. As shown in Fig. 2, a scheduler at the BS chooses the best subset $\mathcal{S}$ of $M$ users and it assigns 


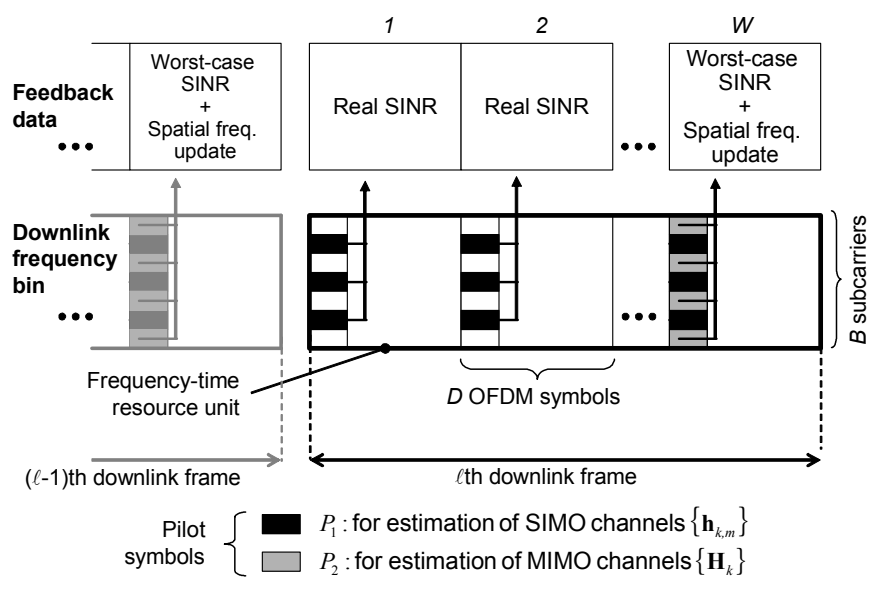

Fig. 1. Overview of the uplink fedback quantities (top) and the downlink frame structure (bottom).

to them $M$ different spatial channels by means of $M$ precoding vectors $\left\{\mathbf{w}_{m}\right\}_{m=1}^{M}$. User selection is performed in each timefrequency unit based on some channel measurements drawn by the MS during a previous training phase and transmitted to the BS through a feedback channel.

In any given time-frequency unit, the $N_{\mathrm{R}} \times 1$ signal received by the $k$ th user $(k=1, \ldots, M)$, on a single subcarrier and within a single OFDM symbol, can be modelled as

$$
\mathbf{y}_{k}=P_{k}^{1 / 2} \underbrace{\mathbf{H}_{k} \mathbf{w}_{k}}_{\mathbf{h}_{k k}} c_{k}+\sum_{m=1, m \neq k}^{M} P_{m}^{1 / 2} \underbrace{\mathbf{H}_{k} \mathbf{w}_{m}}_{\mathbf{h}_{k m}} c_{m}+\mathbf{n}_{k}
$$

where, for the $k$ th user, $\mathbf{H}_{k}$ denotes the $N_{\mathrm{R}} \times N_{\mathrm{T}}$ channel matrix, $\mathbf{w}_{k}$ is the $N_{\mathrm{T}} \times 1$ precoding vector, $P_{k}$ is the transmitted power and $c_{k} \in \mathcal{C}^{(n)}$ is the transmitted symbol with $\mathrm{E}\left[\left|c_{k}\right|^{2}\right]=1$. Since adaptive transmission is used to satisfy a fixed bit error rate (BER), the complex symbol $c_{k}$ can belong to any of the $N$ available modulation sets $\left\{\mathcal{C}^{(n)}\right\}_{n=1}^{N}$. Furthermore, the additive noise $\mathbf{n}_{k}$ is assumed to be zeromean white complex Gaussian with $\mathrm{E}\left[\mathbf{n}_{k} \mathbf{n}_{k}^{\mathrm{H}}\right]=\sigma^{2} \mathbf{I}_{N_{\mathrm{R}}}$. In (1), we defined as $\mathbf{h}_{k m}=\mathbf{H}_{k} \mathbf{w}_{m}$ the $N_{\mathrm{R}} \times 1$ equivalent single input multiple output (SIMO) channel between the BS and the $k$ th MS when the transmitted signal is precoded by $\mathbf{w}_{m}$. The channel response is herein considered as frequency-flat within

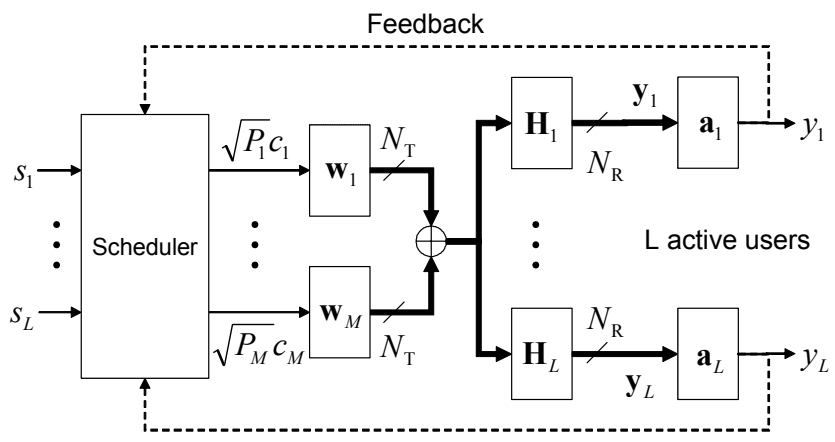

Feedback

Fig. 2. Downlink multiuser MIMO system. the frequency bin, thus the bin size needs to be adequately designed according to the specific propagation environment [9].

As shown in Fig. 2, a spatial filtering $y_{k}=\mathbf{a}_{k}^{H} \mathbf{y}_{k}$ is performed at the receiver side on signal (1) in order to maximize the SINR, according to the MVDR technique. CSI is required at the MS for the evaluation of the MVDR filter but also at the BS for scheduling purposes. Here we assume that channel estimation can be performed by each user from pilot subcarriers included in each burst, as indicated in Fig. 1. More specifically, a first kind of training phase (indicated as $P_{1}$ ) is carried out in each burst by activating at the BS the precoders $\left\{\mathbf{w}_{m}\right\}_{m=1}^{M}$ and estimating the whole set $\left\{\mathbf{h}_{k m}\right\}_{m=1}^{M}$ at the $k$ th MS. A second kind of training phase, needed for the scheduling process and denoted by $P_{2}$, is assumed to be carried out in the last burst of each frame, without use of precoding, to allow the estimation of the MIMO channel matrix $\mathbf{H}_{k}$. Hence, in the following we will assume $\left\{\mathbf{h}_{k m}\right\}_{m=1}^{M}$ and $\mathbf{H}_{k}$ to be known at the $k$ th MS.

As regards CSI at the BS, optimum selection of the user set $\mathcal{S}$ and the corresponding precoding set $\left\{\mathbf{w}_{m}\right\}_{m=1}^{M}$ would require the knowledge of all the MIMO channels $\left\{\mathbf{H}_{m}\right\}_{m=1}^{M}$ and a consequent intensive feedback transmission. In this paper we propose a suboptimal precoding strategy to reduce the feedback. Information to be evaluated at each MS and transmitted on the feedback channel is reduced to a SINR value $\rho_{k}$ after MVDR receiver with the following expression:

$$
\rho_{k}=P_{k} \mathbf{h}_{k k}^{H}\left(\sum_{m=1, m \neq k}^{M} P_{m} \mathbf{h}_{k m} \mathbf{h}_{k m}^{H}+\sigma^{2} \mathbf{I}_{N_{\mathrm{R}}}\right)^{-1} \mathbf{h}_{k k}
$$

and a spatial frequency $f_{k}$ related to the main DOD of the MIMO channel $\mathbf{H}_{k}$. The scheduling procedure based on such measurements consists of two distinct phases:

1) Spatial channel allocation. The user subset $\mathcal{S}$ and the precoders $\left\{\mathbf{w}_{k}\right\}_{k=1}^{M}$, to be maintained during the whole frame, are selected by the scheduler according to the following feedback measurements: a SINR lower bound $\hat{\rho}_{k}$ for (2) based on a worst-case choice of the interference configuration and an estimate $\hat{f}_{k}$ of the spatial frequency for all users (more details can be found in Sections IV-B and II-A respectively). Based on $\left\{\hat{\rho}_{k}\right\}_{k=1}^{M}$, a suboptimal choice for transmission modes is also carried out, while transmission powers $\left\{P_{k}\right\}_{k=1}^{M}$ are uniformly allocated. Finally, within the first burst of the frame, pilot symbols $P_{1}$ are transmitted to the chosen terminals, using the selected precoders (see Fig. 1).

2) Transmission mode selection. Once the precoded channels $\left\{\mathbf{h}_{k m}\right\}_{m=1}^{M}$ have been estimated by the terminals from pilot symbols $P_{1}$, the real SINR measurements $\left\{\rho_{k}\right\}_{k=1}^{M}$ could be evaluated from (2) and be fed back. Transmission powers and transmission modes can be then optimally designed by the scheduler for the remaining $W-1$ bursts.

\section{A. Channel structure}

For the description of each MIMO matrix $\mathbf{H}_{k}$, we adopt the propagation model [6] recalled in Fig. 3. The MS antenna array is assumed to be surrounded by a ring of uniformly 


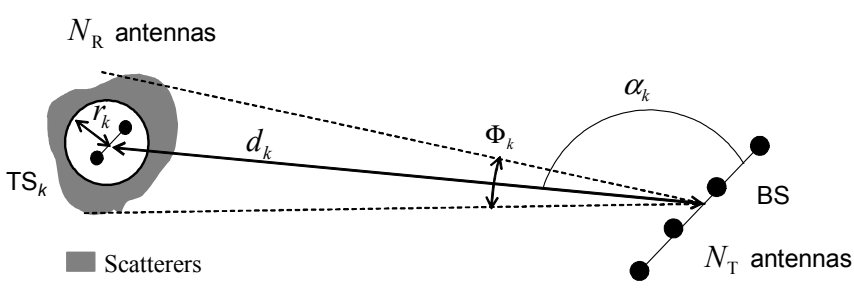

Fig. 3. Spatial structure of the downlink channel.

distributed scatterers of radius $r_{k}$, while the $\mathrm{BS}$, at distance $d_{k} \gg r_{k}$ from the MS, is not surrounded by local scatterers. It follows that the signal to the $k$ th MS is characterized by an angular spread $\Phi_{k} \simeq 2 r_{k} / d_{k}$ around a main DOD that is here denoted by $\alpha_{k}$. The spread $\Phi_{k}$ will in general be smaller than the beamwidth of the array.

Multipath structure, under these conditions, can be approximated by a rank-one channel $\mathbf{H}_{k} \approx \mathbf{v}_{k} \cdot \mathbf{b}^{\mathrm{H}}\left(f_{k}\right)$, where $\mathbf{b}\left(f_{k}\right)$ denotes the $N_{\mathrm{T}} \times 1$ response to the spatial frequency $f_{k}=\cos \left(\alpha_{k}\right)$ for the antenna array at the BS, with elements $\left[\mathbf{b}\left(f_{k}\right)\right]_{m}=\left(1 / \sqrt{N_{T}}\right) \exp \left[-j 2 \pi f_{k}(m-1) \Delta / \lambda\right]$ and $\lambda$ denoting the carrier wavelength. The $N_{\mathrm{R}} \times 1$ vector $\mathbf{v}_{k}$ collects the fading channel gains at the MS array. The main DOD can be estimated at the MS: here it is evaluated from the MIMO matrix $\mathbf{H}_{k}$ (estimated or predicted during previous bursts from pilot symbols $P_{2}$ - figure 1) by a a rank-1 constrained leastsquares optimization:

$\hat{f}_{k}=\arg \min _{f_{k}}\left\|\mathbf{H}_{k}-\mathbf{v}_{k} \mathbf{b}^{\mathrm{H}}\left(f_{k}\right)\right\|^{2}=\arg \max _{f_{k}}\left\{\mathbf{b}^{\mathrm{H}}\left(f_{k}\right) \mathbf{R}_{k} \mathbf{b}\left(f_{k}\right)\right\}$

where $\|\cdot\|$ operator denotes Frobenius norm and $\mathbf{R}_{k}=\mathbf{H}_{k}^{H} \mathbf{H}_{k}$ the instantaneous spatial channel correlation at the BS. We recall that this parameter, once estimated at the MS, needs to be transmitted to the BS for scheduling. Still, since in practical systems the DOD's can be considered as slow-varying among each time-frequency unit, only a limited feedback rate is actually required. In particular, since the frame duration is designed according to the fast-fading parameters, the spatial frequency has to be estimated during the mobile switching on phase, then it may be simply updated every $W$ bursts using a lower number of bits. Section IV-A gives further details on how to perform both the estimate and the update in the most efficient and simplest way.

\section{SCHEDULING AT BS}

This section focuses on the processing to be carried out at the BS. In particular, both the problems of finding the best user subset $\mathcal{S}$ and how to set up the precoders for a specific set of $M$ feedback spatial frequencies have been tackled. A suboptimal solution to the first one is proposed in section III-A. Section III-B deals with the second problem. In the following we restrict our attention to a simplified scheduler with uniform power allocation, from (2) we thus assume $\left\{P_{k}\right\}_{k=1}^{M}=P / M$. The transmission modes $\mathcal{C}^{(n)}$ can be derived from the SINR measurements (2) according to a target BER, as exemplified in Table I for the transmission
TABLE I

IEEE 802.16 TRASMISSION MODES AND REQUIRED SNR RANGES.

\begin{tabular}{||c|c|c|c||}
\hline \hline Modulation & $\begin{array}{c}\text { Coding } \\
\text { rate }\end{array}$ & $\begin{array}{c}\text { SNR } \rho \\
{[\mathrm{dB}]}\end{array}$ & $\begin{array}{c}\text { Spectral efficiency } \gamma \\
{[\text { bit/carrier] }}\end{array}$ \\
\hline \hline QPSK & $1 / 2$ & $9.4-11.2$ & 1 \\
\hline QPSK & $3 / 4$ & $11.2-16.4$ & 1.5 \\
\hline 16-QAM & $1 / 2$ & $16.4-18.2$ & 2 \\
\hline 16-QAM & $3 / 4$ & $18.2-22.7$ & 3 \\
\hline 64-QAM & $2 / 3$ & $22.7-24.4$ & 4 \\
\hline 64-QAM & $3 / 4$ & $>24.4$ & 4.5 \\
\hline \hline
\end{tabular}

modes of the IEEE 802.16-2004 standard [10] and BER $=10^{-6}$ (for additive white Gaussian noise - AWGN - channel). We assume that $F$ scheduler modules are operating independently on the $F$ available frequency bins.

\section{A. User subset selection}

If $L$ users are competing for a specific time-frequency resource, then the scheduler exploits the feedback SINR lower bounds $\left\{\hat{\rho}_{k}\right\}_{k=1}^{L}$ and the spatial frequencies $\left\{\hat{f}_{k}\right\}_{k=1}^{L}$ in order to: 1) find all sets of $M$ users fulfilling a spatial separation constraint (to avoid critical MUI situations); 2) among the selected sets, find the one set that maximizes the whole throughput.

Let $\left\{\mathcal{S}_{i}\right\}_{i=1}^{N_{\mathcal{S}}}$ indicate all the $N_{\mathcal{S}}=L ! /(M !(L-M) !)$ subsets of $M$ users that can be obtained from the whole set of $L$ active terminals. For the $i$ th subset, $\mathcal{S}_{i}=\left\{k_{i, 1}, \ldots, k_{i, M}\right\}$, the corresponding SINR lower bounds and spatial frequency values will be indicated as $\left\{\hat{\rho}_{k_{i, 1}}, \ldots, \hat{\rho}_{k_{i, M}}\right\}$ and $\left\{\hat{f}_{k_{i, 1}}, \ldots, \hat{f}_{k_{i, M}}\right\}$, respectively. For any subset $\mathcal{S}_{i}$ we define the minimum spatial separation between its users as

$$
\mathcal{L}\left(\mathcal{S}_{i}\right)=\min _{\substack{k_{i, \ell}, k_{i, m} \in \mathcal{S}_{i} \\ \ell \neq m}}\left|\hat{f}_{k_{i, \ell}}-\hat{f}_{k_{i, m}}\right| .
$$

We also define the overall throughput as $\gamma\left(\mathcal{S}_{i}\right)=$ $\sum_{m=1}^{M} \gamma\left(\hat{\rho}_{k_{i, m}}\right)$ where $\gamma\left(\hat{\rho}_{k_{i, m}}\right)$ [bit/carrier/user] denotes the spectral efficiency that can be achieved by user $k_{i, m}$ under the worst-case assumption of instantaneous SINR equal to $\hat{\rho}_{k_{i, m}}$. Herein a maximum throughput based scheduling is employed, spectral efficiencies are evaluated from Table I. Nevertheless, the proposed approach could be easily extended in case other throughput measurements and scheduling schemes such as proportional fair scheduling [11] are adopted.

The user subset $\mathcal{S}$ is selected through the following optimization:

$\mathcal{S}=\arg \max _{\mathcal{S}_{i}} \mathcal{L}\left(\mathcal{S}_{i}\right)$ s.t. 1) $\gamma(\mathcal{S})=\max _{\mathcal{S}_{i}} \gamma\left(\mathcal{S}_{i}\right)$, 2) $\mathcal{L}(\mathcal{S})>\beta$.

The two constraints above impose that the solution $\mathcal{S}$ must belong to the set of subsets $\mathcal{S}_{i}$ that: 1) provide the maximum value for the throughput $\gamma\left(\mathcal{S}_{i}\right)$ (calculated based on the SINR lower bounds); 2) guarantee a minimum spatial separation $\beta$ between users. Among these subsets, the optimum is obtained as the one that maximizes the angular separation between users. Notice that the minimum user spacing $\beta$ has to be selected based on the antenna array resolution at the BS [12], 


\section{B. Precoder Computation}

Once the set $\mathcal{S}$ is chosen, appropriate precoding vectors have to be set up for the $M$ selected users according to the feedback spatial frequencies $\hat{\mathbf{f}}=\left[\hat{f}_{1} \cdots \hat{f}_{M}\right]$. Defined as $\mathbf{B}(\hat{\mathbf{f}})=\left[\mathbf{b}\left(\hat{f}_{1}\right) \cdots \mathbf{b}\left(\hat{f}_{M}\right)\right]$ the $N_{\mathrm{T}} \times M$ matrix gathering the BS antenna array responses to the $M$ spatial frequencies, the precoding matrix $\mathbf{W}(\mathbf{f})=\left[\mathbf{w}_{1} \cdots \mathbf{w}_{M}\right]$ is calculated as

$$
\mathbf{W}(\mathbf{f})=\mathbf{B}(\mathbf{f})\left[\mathbf{B}^{\mathrm{H}}(\mathbf{f}) \mathbf{B}(\mathbf{f})\right]^{-1} .
$$

Notice that this choice makes the $m$ th precoder $\mathbf{w}_{m}$ orthogonal to all other vectors $\mathbf{b}\left(\hat{f}_{k}\right)$ within $\mathcal{S}$ for $k \neq m$. Therefore, in the ideal case of null angular-spread at the BS, i.e. $\mathbf{H}_{k}=$ $\mathbf{v}_{k} \cdot \mathbf{b}^{\mathrm{H}}\left(f_{k}\right)$, and for perfect knowledge of user DOD $\left(\hat{f}_{k}=f_{k}\right)$, this orthogonal precoding leads to a null MUI in the MS signal (1). It is understood that perfect interference rejection is no longer guaranteed in more realistic propagation conditions.

\section{CSI FEEDBACK}

As anticipated in Section II, in our framework the channel matrix $\mathbf{H}_{k}$ is assumed to be estimated or predicted [13] [14] by the MS from pilot symbols $P_{2}$ (see Fig. 1). Knowledge of the MIMO matrix is then exploited, as described in the following, to update the spatial frequency $\hat{f}_{k}$ (Section IVA) and compute the SINR lower bound $\hat{\rho}_{k}$ (Section IV-B) to be then transmitted on the feedback channel. A SINR and a spatial frequency computation for each frequency bin should be avoided exploiting interpolation methods [15] to reconstruct all the needed quantities (i.e. SINRs or beamforming vectors) from the knowledge of a minimal subset of the required variables.

\section{A. Spatial frequency estimation}

The estimation for the spatial frequency $f_{k}$ in (3) has to be carried out with no prior information whenever the terminal is turned on. Notice that, under the assumption of quantization of $\hat{f}_{k}$ over $b$ bits, the maximization in (3) requires $16 N_{\mathrm{T}}^{2} 2^{b}$ flops that can be easily reduced to $4\left(N_{\mathrm{T}}-1\right) 2^{b}$ taking into account the symmetric structure of $\mathbf{R}_{k}$.

Since the spatial structure of the multipath channel is assumed to be slowly varying over the frames, the frequency estimate will be updated frame by frame during each spatial allocation phase. A low-complexity algorithm is proposed for the update. The estimate $\hat{f}_{k, \ell-1}$ for the spatial frequency in the $\ell$ th frame is calculated from the previous frame estimate $\hat{f}_{k, \ell-1}$ and the current channel correlation $\mathbf{R}_{k}$ according to:

$$
\hat{f}_{k, \ell}=Q\left[\hat{f}_{k, \ell-1}+\mu \frac{\partial}{\partial f}\left|\mathbf{b}^{\mathrm{H}}(f) \mathbf{R}_{k} \mathbf{b}(f)\right|_{f=\hat{f}_{k, \ell-1}}\right]
$$

where $Q[\cdot]$ is the quantizer operator. The gradient in (7) is herein approximated by exploiting the Hermitian symmetry of $\mathbf{R}_{k}$. It can be shown indeed, by tedious but straightforward algebraic computations, that the following equality holds:

$$
\mathbf{b}^{\mathrm{H}}(f) \mathbf{R}_{k} \mathbf{b}(f) \simeq \Gamma_{0}\left[\mathbf{R}_{k}\right]+\operatorname{Re}\left[\Omega\left(\mathbf{R}_{k}, f\right)\right]
$$

where the first term

$$
\Gamma_{n}\left[\mathbf{R}_{k}\right]=\frac{1}{N_{\mathrm{T}}-i} \sum_{i=1}^{N_{\mathrm{T}}-i}\left[\mathbf{R}_{k}\right]_{i, i+n}
$$

represents the average of the $n$th diagonal elements of matrix $\mathbf{R}_{k}$, while the second one is the real part of the complex quantity:

$$
\Omega\left(\mathbf{R}_{k}, f\right)=\frac{2}{N_{\mathrm{T}}} \sum_{n=1}^{N_{\mathrm{T}}-1} \Gamma_{n}\left[\mathbf{R}_{k}\right] \exp \left(-j 2 \pi f n \frac{\Delta}{\lambda}\right) .
$$

The gradient of (8) can be easily written as

$\frac{\partial}{\partial f} \mathbf{b}^{H}(f) \mathbf{A}_{k} \mathbf{b}(f) \simeq \frac{\partial}{\partial f} \operatorname{Re}\left[\Omega\left(\mathbf{R}_{k}, f\right)\right]=\frac{2 \pi}{\lambda} \Delta \operatorname{Im}\left[\Omega\left(\mathbf{R}_{k}, f\right)\right]$.

Including the term $\frac{2 \pi}{\lambda} \Delta$ into $\mu$ quantity, the tracking equation (7) reduces to:

$$
\hat{f}_{k, \ell}=Q\left[\hat{f}_{k, \ell-1}+\mu \operatorname{Im}\left[\Omega\left(\mathbf{R}_{k}, \hat{f}_{k, \ell-1}\right)\right]\right]
$$

The step-size parameter $\mu$ needs to be chosen according to the velocity of variations of the channel spatial structure.

\section{B. Lower-bound SINR}

During the spatial channel allocation phase the $L$ active terminals are in charge of sending to the BS a measure of their SINR. However, having no knowledge of the $M$ precoders $\left\{\mathbf{w}_{m}\right\}_{m=1}^{M}$, the MS cannot evaluate the exact SINR value $\rho_{k}$ from (2). Hence, we propose to feedback from the $k$ th MS a lower bound $\hat{\rho}_{k}$ corresponding to the worst case of interference configuration. More specifically, recalling the spatial separation constraint in (5) applied by the scheduler, the worst configuration of interferers for the $k$ th user is associated to the set of $\beta$-spaced spatial frequencies $\left\{f_{m}^{(k)}\right\}_{m=1}^{M}$ distributed within the cell sector around the $k$ th value $f_{k}^{(k)}=\hat{f}_{k}$. In particular, for $M$ odd, it can be shown that the set is unique. The corresponding precoding vectors $\left\{\mathbf{w}_{m}\right\}_{m=1}^{M}$ can be computed as in (6) from the selected frequencies $\left\{f_{m}^{(k)}\right\}_{m=1}^{M}$. The precoders are then plugged in (2) together with the power values $\left\{P_{k}\right\}_{k=1}^{M}=P / M$, yielding the SINR lower bound $\hat{\rho}_{k}$.

\section{NUMERICAL RESUltS}

A MIMO downlink radio interface is simulated according to [16] using the system parameters in Table II. Within a single

TABLE II

SYSTEM PARAMETERS AND DOWNLINK RADIO INTERFACE

\begin{tabular}{||l|l||}
\hline \hline Number of Tx and Rx antennas & $N_{T}=4, N_{R}=2$ \\
\hline Antenna spacings & $\Delta=0.5 \lambda$ \\
\hline Max. number of spatially multiplexed streams & $M=3$ \\
\hline Cell sectors & $120 \mathrm{deg}$ \\
\hline Background SNR & $\mathrm{SNR}=18 \mathrm{~dB}$ \\
\hline Minimum spatial separation among users & $20 \mathrm{deg}$ \\
\hline Maximum terminal velocity & $v=80 \mathrm{~km} / \mathrm{h}$ \\
\hline Carrier frequency & $f_{c}=2 \mathrm{GHz}$ \\
\hline Available downlink bandwidth & $B_{w}=5 \mathrm{MHz}$ \\
\hline Subcarrier spacing & $\Delta f=11 \mathrm{kHz}$ \\
\hline OFDM symbol period & $111 \mu \mathrm{s}$ \\
\hline Burst length & $D=4(444 \mu \mathrm{s})$ \\
\hline Frequency bin length & $B=20(200 \mathrm{kHz})$ \\
\hline Maximum number of frequency bins & $F \leq 25$ \\
\hline Downlink frame length & $W=4(1.776 \mu \mathrm{s})$ \\
\hline \hline
\end{tabular}




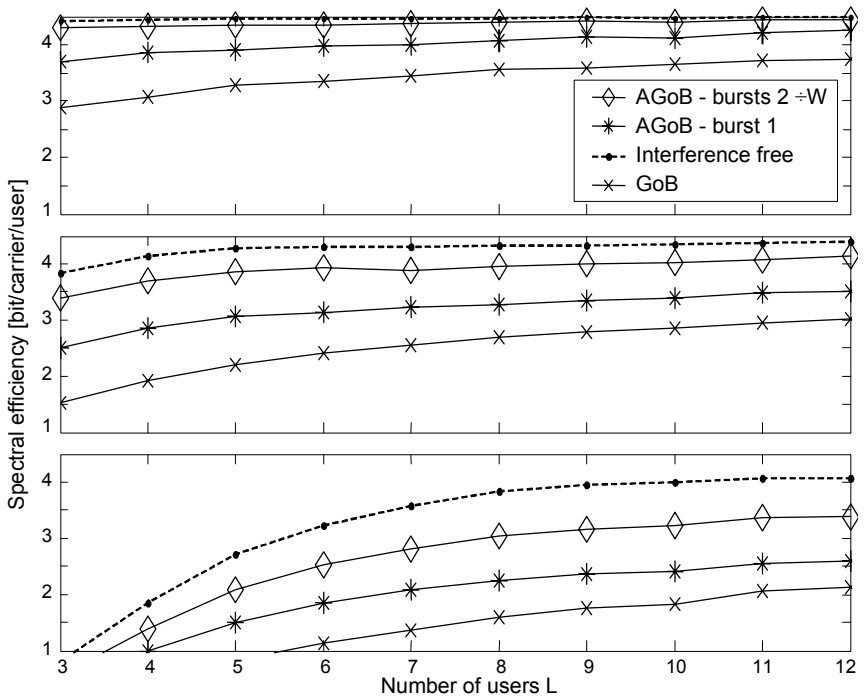

Fig. 4. Average spectral efficiency on each scheduled channel vs. the number $L$ of active users. The adaptive GoB (AGoB) method is compared with the fixed $\mathrm{GoB}$ and the interference-free case. For $\mathrm{AGoB}$, the spectral efficiency is evaluated in either the first or the remaining $W-1$ bursts in the frame.

frequency bin, each burst carries $D \cdot B=80$ symbols; of these, as shown in figure 1, at least $M$ are allocated for the estimation of the precoded channels $\left\{\mathbf{h}_{i k}\right\}_{i=1}^{M}$ (pilot symbols $P_{1}$ in figure 1) and $N_{T}$ for the estimation of the MIMO channel matrix $\mathbf{H}_{k}$ (pilot symbols $P_{2}$ ). Other symbols are used for downlink control messaging. At the BS, spectral efficiencies are computed from SINR measurements as reported in Table I according to the IEEE 802.16-2004 standard [10]. The number of bits used for spatial frequency feedback is $b=6$.

Propagation channels are simulated according to the multipath structure in Section II-A. More specifically, the fading coefficients in matrix $\mathbf{H}_{k}$ are assumed to be Rayleigh distributed, constant within a frame interval but varying from frame to frame. Temporal variations are simulated according to the Clarke model approximated by a second-order autoregressive (AR-2) random process [13]. As regards the spatial structure, users are placed at a fixed distance from the BS with main DOD $\alpha_{k}$ uniformly distributed within the cell sector. Each DOD varies slowly over the frames according to the AR-1 model: $\alpha_{k}(\ell)=\alpha_{k}(\ell-1)+\xi_{k}$, where $\xi_{k} \sim \mathcal{C N}\left(0, \sigma_{\alpha}^{2}\right)$ and $\sigma_{\alpha}=0.5 \mathrm{deg}$. Since multipath channels in urban, suburban, and rural macro-cell are usually characterized by angular spread ranging from $10 \mathrm{deg}$ to $30 \mathrm{deg}$ [5]-[6], we chose for the angular spread $\Phi_{k}$ at the BS a one-sided Gaussian random variable, $\Phi_{k} \sim \mathcal{N}\left(15 \mathrm{deg}, \sigma_{\Phi}^{2}\right), \Phi_{k} \geq 0$, with mean $15 \mathrm{deg}$ and standard deviation $\sigma_{\Phi}=5 \mathrm{deg}$. Spatial correlation of the channel gains at the transmitter and the receiver is modelled according to [6].

Our AGoB solution is compared with the fixed GoB transmission scheme (see D2.7 in [8] for details). In particular, two distinct uniform grids of $M$ beams are switched from frame to frame. Beams are Chebychev tapered and their main directions span uniformly the users' sector. Within the training phase of each frame ( $P_{2}$ symbols in figure 1), the BS
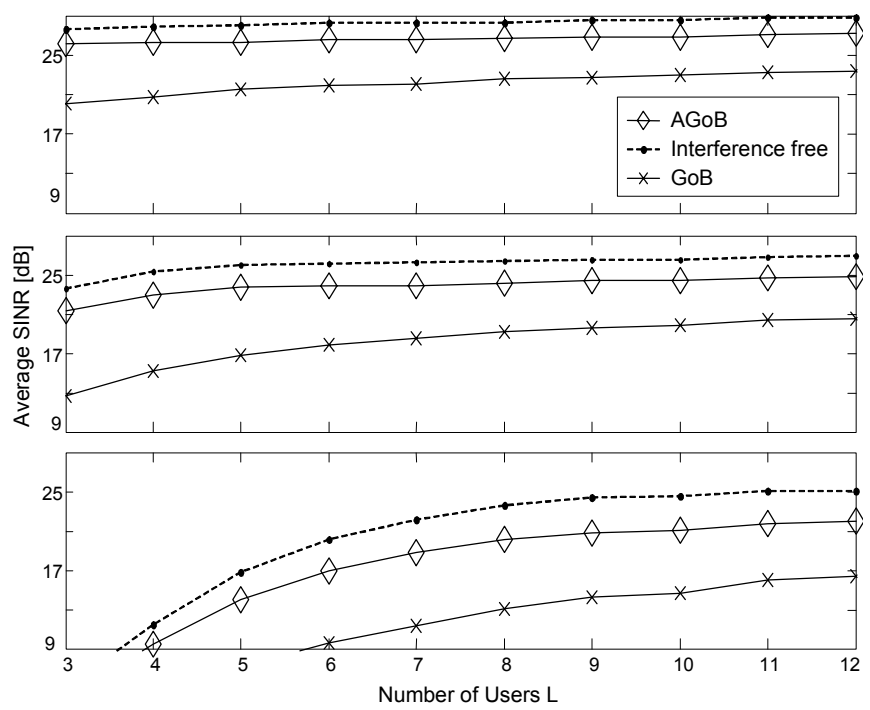

Fig. 5. Average SINR at the MS after MVDR on each scheduled channel vs. the number $L$ of active users. The adaptive GoB (AGoB) method is compared with the fixed $\mathrm{GoB}$ and the interference-free case.

activates the fixed grid of precoders $\left\{\mathbf{w}_{m}\right\}_{m=1}^{M}$ selected for the next frame, to allow the estimation of $\left\{\mathbf{h}_{k m}\right\}_{m=1}^{M}$ by the $k$ th terminal. Precoders are then chosen by the MSs through the maximization of (2) and they are reported to the BS by the transmission of a sequence of $\left\lceil\log _{2} M\right\rceil$ bits. Also SINR measurements (2) have to be fed back to the BS for each frequency bin.

Figure 4 shows the spectral efficiency for each scheduled channel (ordered by SINR value $\hat{\rho}_{k}$ ) averaged over user positions, fading channels and data. Accordingly, figure 5 gives the average SINR at the MS after MVDR. Both plots are drawn versus the number $L$ of active users. Since in most practical cases the number of active users competing for a specific resource unit has a wide and unpredictable range, we considered $L$ values ranging from $L=3$ to $L=12$. For the evaluation of AGoB spectral efficiency and SINR we considered a single OFDM payload symbol within each time-frequency unit, taken either from the first burst (AGoB burst 1) or from any of the remaining $W-1$ bursts (AGoB - burst $2 \div W$ ) of the downlink frame. As shown in figure 4 , when considering the first burst, we found that an average spectral efficiency gain of at least $0.5 \mathrm{bit} / \mathrm{carrier}$ per user (or, equivalently, per channel) can be achieved compared to the GoB solution, for any active users' number $L$. The gain is doubled (at least 1 bit/carrier per user) in case of payload symbol placed in any of the remaining $W-1$ bursts. From figure 5, we can also conclude that a SINR gain of at least $4 \mathrm{~dB}$ can be obtained for each user in any burst of the frame. It is also worth noticing that the performance gains are uniformly distributed among the scheduled channels, for any active users' number $L$. Benefits in spectral efficiency and SINR values are more evident when few users are competing for the same resource unit (i.e., for low $L$ ).

MUI reduction capability of the proposed AGoB system 


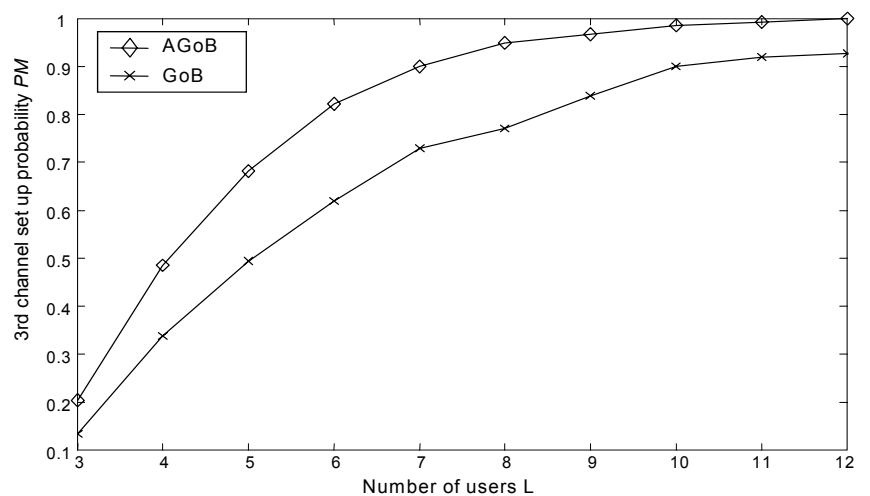

Fig. 6. 3rd set up channel probability $p_{M}$ vs. the number of users $L$ for both the AGoB and the fixed GoB approaches $(M=3)$.

with the spectral efficiency (figure 4) and the SINR (figure 5 ) of the interference-free case (dashed lines). This ideal case refers to a scenario where $M$ channels can be set up for the same users selected by AGoB, but with null MUI and spectral efficiencies $\gamma\left(\rho_{k}^{\text {(id) }}\right)$ drawn from the signal-to-noise ratio (SNR) $\rho_{k}^{(\mathrm{id})}=P_{k}\left\|\mathbf{H}_{k}\right\|^{2} / \sigma^{2}$ for $k=1, \ldots, M$ (as for a single-user AWGN channel). It can be shown that this perfect interference rejection could be achieved by (6) only in case of perfect knowledge of each user DOD $\left(\hat{f}_{k}=f_{k}\right)$ and null angular spread $\left(\Phi_{k}=0\right)$. Figure 5 shows that in more realistic channel conditions the SINR loss experienced by AGoB with respect to this ideal case is $2 \mathrm{~dB}$ only, for any active users' number $L$. From this result we can conclude that the proposed scheduling and precoding approach at the BS, jointly with MVDR filtering at the MS, is effectively able to reduce the multiple-access interference, thus increasing the overall spectral efficiency.

Before closing this Section, we consider a final performance comparison based on the probability $p_{M}$ that all the $M$ channels can be reliably set up: $p_{M}=p\left[\gamma\left(\min _{k \in \mathcal{S}}\left(\hat{\rho}_{k}\right)\right) \geq 1\right]$. This probability is evaluated in figure 6 for $\mathrm{AGoB}$ and $\mathrm{GoB}$, for $M=3$ and for varying number $L$ of active users. The comparison between the two methods shows that, in order to schedule $M$ users for the same time-frequency unit, AGoB requires a total number of terminals that is lower compared to the fixed GoB approach: for $L=7 \mathrm{AGoB}$ guarantees a third channel to be set up with probability larger than $90 \%$, while GoB needs an higher $L$ value to achieve the same reliability. In particular, whenever limited $L$ values are considered, an higher fraction of active users can be served: as a consequence AGoB leads to a significant increase also in coverage capabilities.

\section{CONCLUSION}

An SDMA technique for a MIMO OFDM FDD system has been proposed. It has been shown that the same time-frequency unit can be scheduled to several users in the same coverage area leading to a more efficient use of channel resources. Although users' subset at the BS is suboptimally designed according to partial CSI in the form of spatial frequencies and SINR lower bounds, significant benefits compared to the fixed GoB approach could be attained exploiting the channel spatial structure and the precoder adaptation. Such high throughput gains are uniformly distributed among each scheduled channel, as a consequence they justify a reasonable complexity growth at the receiver, due to both channel (and spatial frequency) estimation processing, and the need for a greater number of pilot symbols. Both clever scheduling based on spatial separation among users, precoding processing at the transmitter and spatial MVDR filtering at the receiver have a substantial impact on the MUI reduction. Finally, significant benefits also in coverage capabilities could be achieved exploiting precoders adaptation.

\section{REFERENCES}

[1] I. Koutsopoulos, L. Tassiulas, "Adaptive resource allocation in SDMAbased wireless broadband networks with OFDM signaling," Proc. IEEE INFOCOM 2002, vol. 3, pp. 1376-1385, June 2002.

[2] J. Kim, J. Cioffi, "Spatial multiuser access with antenna diversity using singular value decomposition," IEEE Proc. ICC 2000, vol. 3, pp. $1253-$ 1257 , June 2000

[3] Y.J. Zhang, K.B. Letaief, "An efficient resource-allocation scheme for spatial multiuser access in MIMO/OFDM Systems," IEEE Trans. Commun., vol. 53, no. 1, pp. 107-116, Jan. 2005.

[4] G. Primolevo, O. Simeone and U. Spagnolini, "Channel aware scheduling for broadcast MIMO systems with orthogonal linear precoding and fairness constraints," IEEE Proc. ICC 2005, vol. 4, pp. 2749-2753, May 2005.

[5] U. Martin, "Spatio-temporal radio channel characteristics in urban macrocells," IEE Proc. Radar, Sonar and Navigation, vol. 145, pp. 4249, Feb. 1998.

[6] A. Abdi, M. Kaveh, "A space-time correlation model for multielement antenna systems in mobile fading channels," IEEE J. Select. Areas Commun., vol. 20, no. 3, pp. 550-560, April 2002.

[7] D.J. Love, R.W. Heath Jr., "Limited feedback precoding for spatial multiplexing systems," IEEE Proc. GLOBECOM 2003, vol. 4, pp. 18571861, Dec. 2003

[8] EU Integrated Project WINNER Phase I, "Assessment of advanced beamforming and MIMO technologies," Deliverable D2.7. "Assessment of adaptive transmission technologies." Deliverable D2.4. Both available at https://www.ist-winner.org.

[9] W. Wang, T. Ottosson, M. Sternad, A. Ahlén and A. Svensson, "Impact of multiuser diversity and channel variability on adaptive OFDM," IEEE Proc. VTC 2003-Fall. vol. 1, pp. 547-551, Oct. 2003.

[10] Standard specifications 802.16-2004, available at http://ieee802.org/16.

[11] T. Park, O.S. Shin and K.B. Lee, "Proportional fair scheduling for wireless communication with multiple transmit and receive antennas," IEEE Proc. VTC 2003-Fall, vol. 3, pp. 1573-1577, Oct. 2003.

[12] U. Spagnolini, "A Simplified Model to Evaluate the Probability of error in DS-CDMA systems with adaptive arrays," IEEE Trans. Wireless Commun., vol. 3, no. 2, pp. 578-587, March 2004.

[13] M. Sternad, D. Aronsson, "Channel estimation and prediction for adaptive OFDM downlinks," IEEE Proc. VTC2003-Fall, vol. 2, pp. 12831287, Oct 2003.

[14] M. Cicerone, O. Simeone and U. Spagnolini, "Channel estimation for MIMO-OFDM systems by modal analysis/ filtering," submitted to IEEE Trans. Commun.

[15] J. Choi, R. W. Heath, Jr, "Interpolation based transmit beamforming for MIMO-OFDM with limited feedback," IEEE Trans on Signal Processing, vol 53, no 11, pp 4125- 4135, Nov 2005.

[16] T. Ottosson, M. Sternad, A. Ahlén and A. Svensson, "Attaining both coverage and high spectral efficiencies with adaptive OFDM downlinks," IEEE Proc. VTC 2003-Fall. vol. 1, pp. 2486-2490, Oct 2003. 